\title{
Avaliação da Inversão Geofísica pela Decomposição por Valores Singulares num Experi- mento Numérico em Gravimetria 2D Linear
} Silvia Lorena Bejarano Bermúdez (IGEO/UFBA), Amin Bassrei (IGEO/CPGG/UFBA)

Copyright 2012, SBGf - Sociedade Brasileira de Geofísica Este texto foi preparado para a apresentação no $V$ Simpósio Brasileiro de Geofísica, Salvador, 27 a 29 de novembro de 2012. Seu conteúdo foi revisado pelo Comitê Técnico do V SimBGf, mas não necessariamente representa a opinião da SBGf ou de seus associados. É proibida a reprodução total ou parcial deste material para propósitos comerciais sem prévia autorização da SBGf.

\section{RESUMO}

Neste trabalho avaliamos a qualidade da solução em problemas inversos em Geofísica de Exploração quando utilizado o método da Decomposição por Valores Singulares, ou SVD do inglês singular value decomposition. O problema considerado foi a inversão de dados gravimétricos $2 \mathrm{D}$, caso linear, com uso da informação a priori e com a contaminação dos dados gravimétricos por ruído gaussiano. No exemplo com dados sintéticos, para a modelagem e posterior inversão, a região de interesse foi discretizada em blocos 2D uniformes, considerando constante a densidade em cada bloco. As inversões foram efetuadas com diferentes quantidades de valores singulares, onde em cada situação a quantidade de valores singulares foi escolhida em função do número de condição associado. O número ótimo utilizado foi entorno de 50 valores singulares de um conjunto de 60 , isto foi escolhido por meio dos quatro critérios de seleção de valores singulares, os quais são dados pela energia, a entropia, o erro do modelo e o erro do dado. Para avaliar a qualidade da inversão foram empregadas duas abordagens. Na primeira abordagem foi analizado o comportamento da matriz de resolução de dado e a matriz de resolução de modelo, para os diferentes números de valores singulares empregados. Observouse que a maioria dos elementos da diagonal prinicipal de cada matriz se aproximam de 1 quando se utiliza o número ótimo de valores singulares, o que muda é se os dados estão contaminados ou não pelo ruído gaussiano. A diagonal principal da matriz de resolução de modelo pode ser plotada em forma de matriz e fornece um claro indicador da qualidade da inversão para cada elemento do vetor de parâmetros de modelo estimado. No segundo critério se utiliza os conceitos de vetores complementares, tanto para os parâmetros de modelo como para os parâmetros de dado. A soma da solução estimada com a solução estimada complementar fornece um terceiro vetor ou imagem, que também permite ava- liar a qualidade da inversão em cada elemento do vetor de parâmetros de modelo estimado.

\section{INTRODUÇÃO}

Os geofísicos dedicam seus esforços para obter a solução do problema inverso, mas em geral pouca atenção é dada no tocante à crítica da solução. Em outras palavras, embora a interpretação dos resultados é feita à luz da realidade geológica, pouca ênfase é dada a uma análise quantitativa da solução do problema inverso. $\mathrm{O}$ objetivo principal do presente trabalho é apresentar diferentes metodologias para avaliar a qualidade da inversão em um problema inverso com dados sintéticos em gravimetria $2 \mathrm{D}$ linear.

O problema principal na interpretação gravimétrica ou em qualquer outro método de prospecção geofísica é poder identificar o possível corpo anômalo em subsuperfície a partir de observações em superfície. No presente trabalho efetuamos uma discretização da região em subsuperfície em blocos 2D retangulares com dimensões conhecidas. Para tanto utilizamos a abordagem de de Last e Kubik (1983). Considere o problema $\mathbf{d}=G \mathbf{m}$, onde d é o vetor de parâmetros de dado, que aqui no caso são os valores do campo gravitacional, m é o vetor de parâmetros do modelo correspondente aos contrastes de densidades (em relação ao meio de referência), e os elementos da matriz $G$ são determinados pela geometria dos blocos e sua relação com cada ponto de observação. As simulações de inversão foram feitas com dados livres de ruído assim como com dados contaminados por ruído gaussiano. O vetor $\mathbf{d}$ expresso acima é o vetor observado, isto é, é expresso como $\mathbf{d}^{o b s}$.

O procedimento inverso foi executado pela decomposição por valores singulares, ou SVD do inglês singular value decomposition. Por definição SVD é uma técnica de decomposição de matrizes que consiste em representar qualquer matriz $G_{N \times M}$ na forma $G=U \Sigma V^{T}$, onde $U_{M \times M}$ e $V_{N \times N}^{T}$ são matrizes ortogonais e $\Sigma_{M \times N}$ é a matriz diagonal que contém os valores singulares.

A utilização de modelos sintéticos é necessária para que possamos determinar o erro, isto é, a diferença entre o vetor de parâmetros de modelo verdadeiro $\mathbf{m}^{\text {ver }}$ (modelo sintético) e o vetor de parâmetros de modelo estimado $\mathbf{m}^{e s t}$. Este último vetor está associado ao vetor de parâmetros de dado calculado, representado por $\mathbf{d}^{\text {cal }}$. 
Num conjunto de dados reais não poderíamos fazer tal análise, já que não conhecemos o que realmente há em subsuperfície. Em outras palavras, para um caso real podemos determinar apenas o vetor de parâmetros de modelo estimado.

Em SVD tem-se que evitar os valores singulares pequenos, para não comprometer os resultados, pois estes valores agem como se fossem ruídos. Portanto, se utilizou o critério dos valores singulares os quais são dados pela energia, entropia, erro do modelo e erro do dado, para se fazer uma estimativa do número ideal de valores singulares utilizados na inversão segundo José Silva e Amin Bassrei (2007). Os estimadores de erro que usamos neste trabalho são:

$$
e_{\mathbf{m}}=\frac{\sqrt{\sum_{i=1}^{N}\left(m_{i}^{v e r}-m_{i}^{e s t}\right)^{2}}}{\sqrt{\sum_{i=1}^{N}}\left(m_{i}^{e s t}\right)^{2}} \times 100
$$

que mede a diferença entre o modelo verdadeiro e o estimador, e

$$
e_{\mathbf{d}}=\frac{\sqrt{\sum_{j=1}^{M}\left(d_{j}^{c a l}-d_{j}^{o b s}\right)^{2}}}{\sqrt{\sum_{i=1}^{N}}\left(d_{j}^{o b s}\right)^{2}} \times 100 .
$$

que mede a diferença entre o dado observado e o dado calculado.

Usamos nesse trabalho dois critérios. O primeiro critério é a matriz de resolução, conforme definido em Jackson (1972). Quanto mais próxima for a matriz resolução da matriz identidade melhor será a qualidade da inversão. No segundo critério, consideramos que $\mathbf{m}$ tem um vetor complementar $\mathbf{m}^{C}$ no qual as somas destes dois vetores é um terceiro vetor. A soma destes dois vetores será designada por w, e será constante se o problema inverso for exato. Esse critério foi sugerido por Barbieri (1974).

\section{MODELAGEM EM GRAVIMETRIA}

Para se efetuar a modelagem gravimétrica direta a área de interesse é discretizada em blocos $2 \mathrm{D}$ retangulares com dimensões conhecidas, admitindo que o vetor de parâmetros de modelo $\mathbf{m}$ é a densidade $\rho$. O valor de $\rho$ é constante para cada bloco. Como a densidade pode variar de bloco para bloco, temos que a densidade será função das coordenadas $x$ e $z$, isto é, $\rho=\rho(x, z)$.

A modelagem direta é calculada pela seguinte equação linear: $\mathbf{d}=G \mathbf{m}$, onde: $\mathbf{d}$ é o vetor de $M$ elementos composto pelos valores do campo gravitacional $g, G_{M \times N}$ é a matriz que expressa a geometria do problema, onde $M$ é o número total de observações ou de estações e $N$ é o número de blocos na qual a área em estudo foi discretizada. Finalmente, $\mathbf{m}$ é o vetor de parâmetros de modelo contendo $N$ elementos, no caso aqui expresso pela densidade $\rho$.
Para o trabalho em questão, onde empregamos a modelagem de estruturas bidimensionais, a expressão para o elemento $a_{i j}$ da matriz $G$ é dada por Last e Kubik (1983) e Bassrei, A. (1993):

$$
\begin{aligned}
a_{i j}= & 2 \gamma\left[\left(x_{i}-x_{j}+d / 2\right) \log \left(r_{2} r_{3} / r_{1} r_{4}\right)+\right. \\
& d \log \left(r_{4} / r_{3}\right)-\left(z_{j}+h / 2\right)\left(\theta_{4}-\theta_{2}\right)+ \\
& \left.\left(z_{j}-h / 2\right)\left(\theta_{3}-\theta_{1}\right)\right]
\end{aligned}
$$

onde: $r_{1}^{2}=\left(z_{j}-h / 2\right)^{2}+\left(x_{i}-x_{j}+d / 2\right)^{2}$,

$r_{2}^{2}=\left(z_{j}+h / 2\right)^{2}+\left(x_{i}-x_{j}+d / 2\right)^{2}$,

$r_{3}^{2}=\left(z_{j}-h / 2\right)^{2}+\left(x_{i}-x_{j}-d / 2\right)^{2}$,

$r_{4}^{2}=\left(z_{j}+h / 2\right)^{2}+\left(x_{i}-x_{j}-d / 2\right)^{2}$,

$\theta_{1}=\arctan \left(x_{i}-x_{j}+d / 2\right) /\left(z_{j}-h / 2\right)$,

$\theta_{2}=\arctan \left(x_{i}-x_{j}+d / 2\right) /\left(z_{j}+h / 2\right)$,

$\theta_{3}=\arctan \left(x_{i}-x_{j}-d / 2\right) /\left(z_{j}-h / 2\right)$,

$\theta_{4}=\arctan \left(x_{i}-x_{j}-d / 2\right) /\left(z_{j}+h / 2\right)$.

Os elementos acima podem ser visualizados na Figura 1. Note-se que uma estrutura bidimensional irregular pode ser aproximada pela superposição de muitos retângulos pequenos de densidade constante.

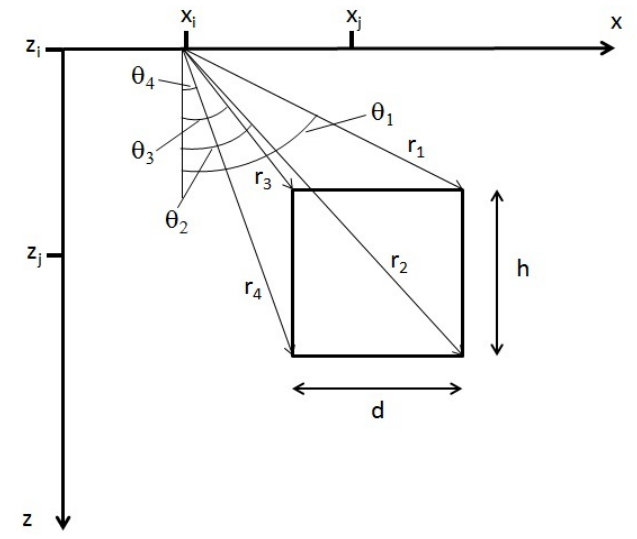

Figura 1: Representação da estrutura de blocos para o cálculo dos elementos da matriz gravimétrica.

O modelo sintético utilizado pode ser observado na $\mathrm{Fi}$ gura 2, na qual se destacam dois corpos anômalos dentro de um meio homogêneo. Um corpo tem densidade de $1,5 \mathrm{~g} / \mathrm{cm}^{3}$ e o outro tem $2,5 \mathrm{~g} / \mathrm{cm}^{3}$. O meio homogêneo tem densidade de $2 \mathrm{~g} / \mathrm{cm}^{3}$. Portanto, o modelo escolhido caracteriza uma anomalia gravimétrica negativa e outra positiva. A região de interesse foi discretizada em 60 blocos, onde cada bloco tem um comprimento de $1 \mathrm{~km}$ e de altura $500 \mathrm{~m}$. Como resultado as dimensões totais da região de interesse são $10 \mathrm{~km}$ de extensão horizontal e $3 \mathrm{~km}$ de profundidade a partir da superfície.

\section{SIMULAÇÕES COMPUTACIONAIS}

Para a inversão SVD o campo gravitacional foi calculado para um problema determinado, com 60 pontos da superfície, isto é, o número de equações é igual ao número de incógnitas. Posteriormente, foi analisado o problema sobredeterminado, no caso com 90 pontos da superfície, 


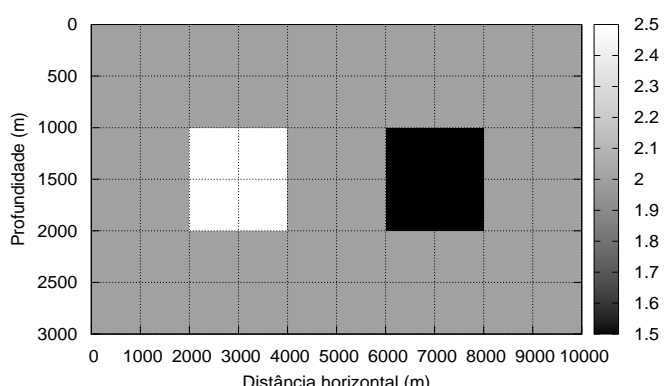

Figura 2: Modelo utilizado para testar a inversão. A escala de cores fornece as densidades, em $\mathrm{g} / \mathrm{cm}^{3}$.

onde agora existem mais equações que incógnitas. Estas inversões foram realizadas em quatro situações diferentes, onde em cada situação mudava a quantidade utilizada de valores singulares. Portanto, em cada situação havia um determinado número de condição $N C$. Esse indicador é expresso pela relação $N C=\sigma_{\max } / \sigma_{\min }$, onde $\sigma_{\max }$ é constante e $\sigma_{\min }$ é diferente em cada situação. Também realizamos simulações com dados livres de ruído, assim como com dados contaminados por ruído gaussiano. A adição de ruído se deu pela expressão $d_{j}^{*}=d_{j}\left(1+\alpha r_{j}\right)$, onde $d_{j}^{*}$ representa o $j$-ésimo elemento do vetor de parâmetros de dado contaminado com ruído, $d_{j}$ representa o $j$-ésimo elemento do vetor de parâmetros de dado livre de ruído, $\alpha$ é a amplitude de ruído aplicado e $r_{j}$ é o $j$-ésimo elemento de uma sequência quase aleatória de números. Foram realizadas muitas simulações cujos resultados estão condensados na Tabela 1. Por limitação de espaço mostramos as figuras de algumas simulações.

Nas Figuras 3 e 4 pode-se observar a inversão quando foram utilizados 50 valores singulares, para o caso determinado e sobredeterminado, respectivamente. Para outras quantidades de valores singulares os resultados podem ser vistos na Tabela 1 . Na mesma Tabela podese observar também como variam os valores percentuais do erro do modelo $e_{\mathbf{m}}$, Equação 1, e do erro de dado $e_{\mathbf{d}}$, Equação 2, à medida que variamos o número de valores singulares e o valor de $\alpha$. Pode-se constatar que ao aumentar o nível de ruído, o erro $e_{\mathbf{m}}$ cresce de forma não-linear. Quando o valor do erro se mostra desproporcional optamos em colocar um traço na coluna de erros do modelo. No caso do erro dos parâmetros de dado $e_{\mathbf{d}}$ observou-se que quando se aumenta o número de valores singulares diminui o valor desse erro. Também quando se diminui o valor de $\alpha$ o valor do erro $e_{\mathbf{d}}$ decresce.

\section{MATRIZES DE RESOLUÇÃO}

Conforme mencionado na Introdução para efetuarmos uma análise quantitativa da solução do problema inverso utilizamos o critério da matriz de resolução. Quanto

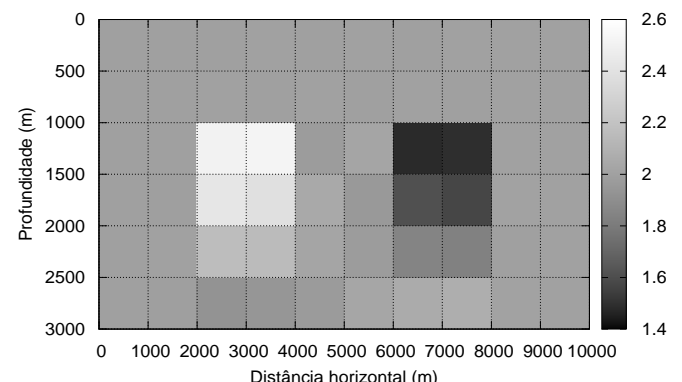

Figura 3: Simulações com o modelo no caso determinado com 50 valores singulares e um $e_{\mathbf{m}}=2,62 \%$.

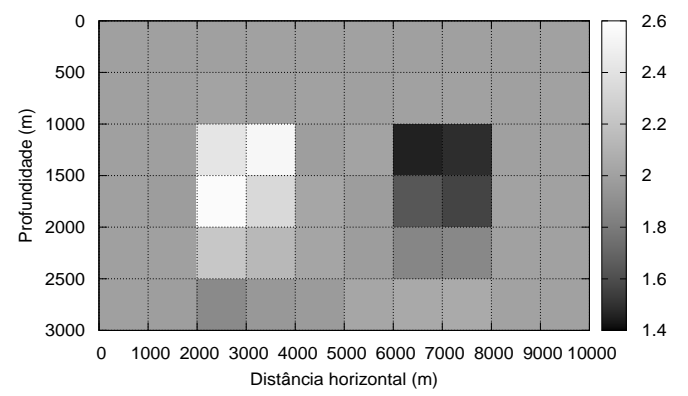

Figura 4: Simulações com o modelo no caso sobredeterminado com 50 valores singulares e um $e_{\mathbf{m}}=2,85 \%$.

mais próxima for a matriz de resolução da matriz identidade melhor será a qualidade da inversão Jackson (1972). Existem duas matrizes de resolução, a matriz de resolução do modelo é:

$$
\begin{aligned}
R_{m} & =G^{+} G \\
& =V \Sigma^{+} U^{T} U \Sigma V^{T} \\
& =V \Sigma^{+} \Sigma V^{T}
\end{aligned}
$$

Por seu turno, a matriz de resolução de dado é:

$$
\begin{aligned}
R_{d} & =G G^{+} \\
& =U \Sigma V^{T} V \Sigma^{+} U^{T} \\
& =U \Sigma \Sigma^{+} U^{T}
\end{aligned}
$$

Nas Figuras 5 e 6, observa-se os resultados da matriz de resolução de dado, com 50 valores singulares, para o problema determinado e sobredeterminado, respectivamente. Pode-se notar que nos dois casos à medida que o número de valores singulares aumenta, os elementos da diagonal da matriz de resolução de dados aproximamse de 1 , significando uma boa inversão. Fazendo uma comparação entre as Figuras observa-se que os valores da diagonal principal da matriz de resolução de dados para um problema determinado, são mais próximos de 


\begin{tabular}{|c|c|c|c|c|c|c|c|}
\hline \multirow{2}{*}{$\alpha$} & Valores Singulares & \multicolumn{3}{|c|}{ Determinado } & \multicolumn{3}{c|}{ Sobredeterminado } \\
\cline { 2 - 8 } & & $e_{\mathbf{m}}(\%)$ & $e_{\mathbf{d}}(\%)$ & $e_{\text {diag }}(\%)$ & $e_{\mathbf{m}}(\%)$ & $e_{\mathbf{d}}(\%)$ & $e_{\text {diag }}(\%)$ \\
\hline 0,001 & 14 & 8,36 & $1,18 \times 10^{-2}$ & 66,11 & 8,37 & $1,37 \times 10^{-2}$ & 66,11 \\
\cline { 2 - 8 } & 26 & 9,02 & $8,54 \times 10^{-3}$ & 44,40 & 8,48 & $1,0 \times 10^{-3}$ & 44,41 \\
\cline { 2 - 8 } & 38 & - & $4,17 \times 10^{-3}$ & 26,19 & - & $8,61 \times 10^{-3}$ & 26,19 \\
\cline { 2 - 8 } & 50 & - & $4,17 \times 10^{-3}$ & 26,19 & - & $8,61 \times 10^{-3}$ & 26,19 \\
\hline 0,005 & 14 & 10,31 & 0,10 & 65,73 & 8,38 & $5,52 \times 10^{-2}$ & 66,11 \\
\cline { 2 - 8 } & 26 & - & $8,40 \times 10^{-2}$ & 44,83 & 22,9 & $4,99 \times 10^{-3}$ & 44,41 \\
\cline { 2 - 8 } & 38 & - & $6,36 \times 10^{-2}$ & 25,91 & - & $4,31 \times 10^{-3}$ & 26,19 \\
\hline \multirow{4}{*}{0,01} & 50 & - & $1,75 \times 10^{-2}$ & 9,22 & - & $4,03 \times 10^{-3}$ & 9,50 \\
\cline { 2 - 8 } & 14 & 8,38 & 0,10 & 66,11 & 8,42 & 0,11 & 66,11 \\
\cline { 2 - 8 } & 26 & - & $8,49 \times 10^{-2}$ & 44,40 & - & $9,98 \times 10^{-2}$ & 44,41 \\
\cline { 2 - 8 } & 38 & - & $4,17 \times 10^{-2}$ & 26,19 & - & $8,61 \times 10^{-2}$ & 26,19 \\
\hline 0,05 & 50 & - & $1,79 \times 10^{-2}$ & 9,06 & - & $8,62 \times 10^{-2}$ & 9,50 \\
\cline { 2 - 8 } & 14 & 8,89 & 0,52 & 66,10 & 9,55 & 0,53 & 66,10 \\
\cline { 2 - 8 } & 26 & - & 0,42 & 44,10 & - & 0,49 & 44,40 \\
\cline { 2 - 8 } & 38 & - & 0,21 & 26,19 & - & 0,43 & 26,19 \\
\hline 0,1 & 50 & - & $8,94 \times 10^{-2}$ & 9,06 & - & 0,40 & 9,50 \\
\cline { 2 - 8 } & 14 & 10,33 & 1,04 & 66,11 & 12,4 & 1,06 & 66,10 \\
\cline { 2 - 8 } & 26 & - & 0,84 & 44,40 & - & 0,99 & 44,40 \\
\cline { 2 - 8 } & 38 & - & 0,42 & 26,19 & - & 0,85 & 26,19 \\
\hline \multirow{4}{*}{0,0} & 50 & - & 0,18 & 9,06 & - & 0,80 & 9,50 \\
\cline { 2 - 8 } & 14 & 8,40 & $6,79 \times 10^{-2}$ & 66,11 & 8,40 & $6,77 \times 10^{-3}$ & 66,11 \\
\cline { 2 - 8 } & 26 & 7,29 & $8,38 \times 10^{-2}$ & 44,40 & 7,29 & $6,27 \times 10^{-5}$ & 44,24 \\
\cline { 2 - 8 } & 38 & 3,03 & $1,59 \times 10^{-2}$ & 26,19 & 3,02 & $6,48 \times 10^{-7}$ & 26,76 \\
\cline { 2 - 8 } & $5,91 \times 10^{-2}$ & 9,06 & 2,84 & $2,52 \times 10^{-3}$ & 10,47 \\
\hline
\end{tabular}

Tabela 1: Porcentagem de erro do modelo $e_{\mathbf{m}}$, erro do dado $e_{\mathbf{d}}$ e erro $e_{\text {diag }}$.

1 do que a diagonal principal da matriz de resolução de dados para um problema sobredeterminado.

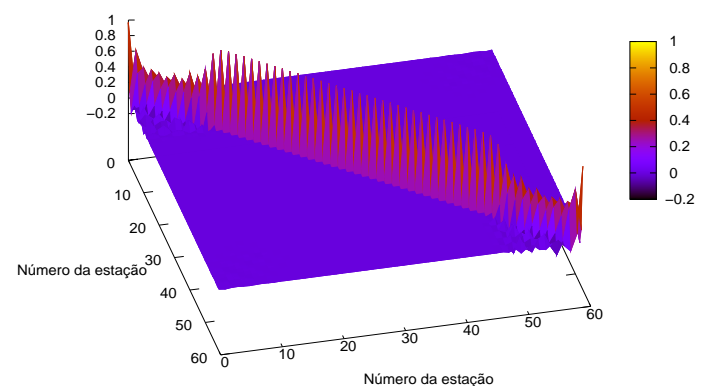

Figura 5: Matriz de resolução de dados, no caso determinado, com 50 valores singulares. A escala de cores fornece a amplitude adimensional.

Nas Figuras 7 e 8 pode ser observado a matriz de resolução de modelo, com 50 valores singulares, para um problema determinado e sobredeterminado, respectivamente. Similarmente, a matriz de resolução de modelo se aproxima mais da matriz identidade quando aumentamos os valores singulares. Fazendo uma comparação entre as figuras se observa que no problema determi-

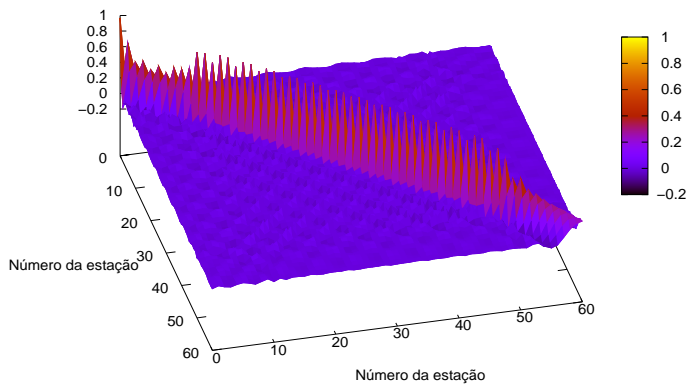

Figura 6: Matriz de resolução de dados, no caso sobredeterminado, com 50 valores singulares. A escala de cores fornece a amplitude adimensional.

nado a matriz de resolução foi muito mais próxima de 1.

Se realizou um estudo dos valores da diagonal principal da matriz de resolução de modelos, com o propósito de observar a qualidade da inversão à medida que variamos os valores singulares. No caso de 50 valores singulares os resultados podem ser vistos nas Figuras 9 e 10. Nestas Figuras visualiza-se o resultado da inversão 


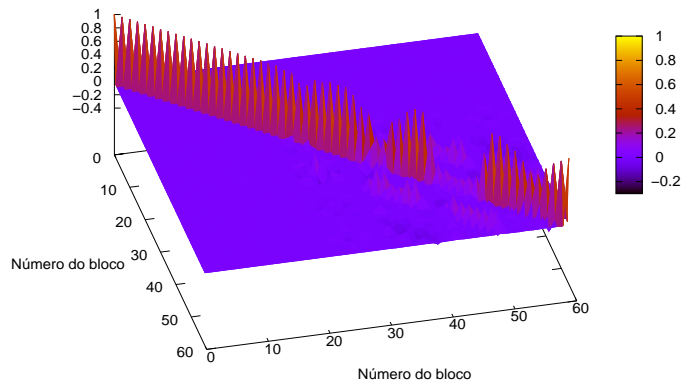

Figura 7: Matriz de resolução de modelo, no caso determinado, com 50 valores singulares. A escala de cores fornece a amplitude adimensional.

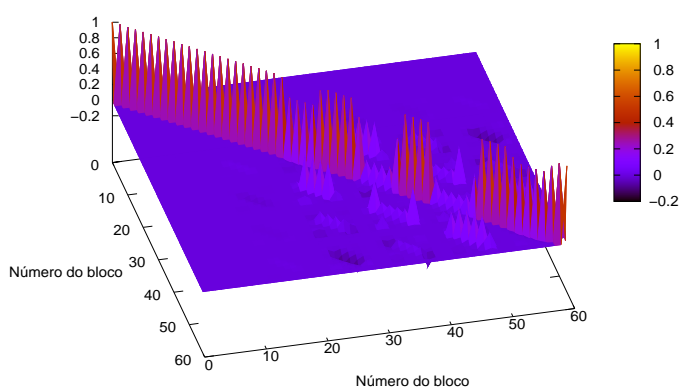

Figura 8: Matriz de resolução de modelo, no caso sobredeterminado, com 50 valores singulares. A escala de cores fornece a amplitude adimensional.

para cada um dos blocos que representam o meio discretizado. Quando o valor dos blocos nas Figuras 9 e 10 se aproxima de 1 , temos que a inversão teve um bom desempenho, e os resultados deixam a desejar quando o valor do bloco diverge de 1 . A Tabela 1 informa ainda os erros percentuais médios da diagonal principal da matriz de resolução de modelo $e_{\text {diag }}$, os quais foram calculados pela Equação 4. Nota-se que para cada nível de ruído esse erro diminui à medida que se utiliza mais valores singulares. Nas Figuras 9 e 10, que representam as simulações sem adição de ruído, o erro percentual foi de $e_{\text {diag }}=9,06 \%$ para o caso determinado e $e_{\text {diag }}=10,47 \%$ para o caso sobredeterminado.

$$
e_{\text {diag }}=\frac{\sum_{i=1}^{N}\left(1-\operatorname{diag}_{i}\right)^{2}}{N} \times 100
$$

\section{MÉTODO DE BARBIERI}

O segundo método foi sugerido por Barbieri (1974) e utilizado em tomografia médica.Considere que $\mathbf{m}$, solução de um problema inverso, tem um vetor complementar $\mathbf{m}^{C}$, de modo que a soma destes dois vetores é um vetor constante dado por w. Podemos trabalhar tanto

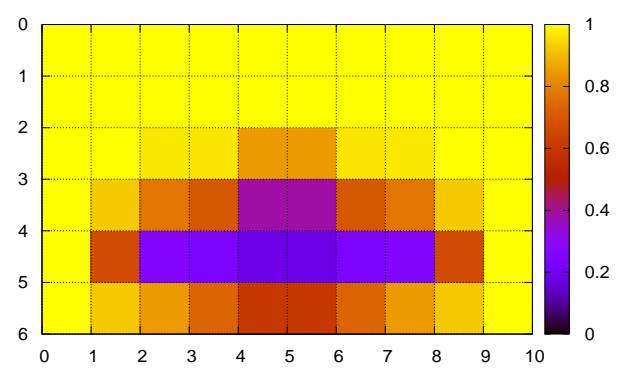

Figura 9: Diagonal principal da matriz de resolução de modelo, caso determinado, com 50 valores singulares. A escala de cores fornece a amplitude adimensional.

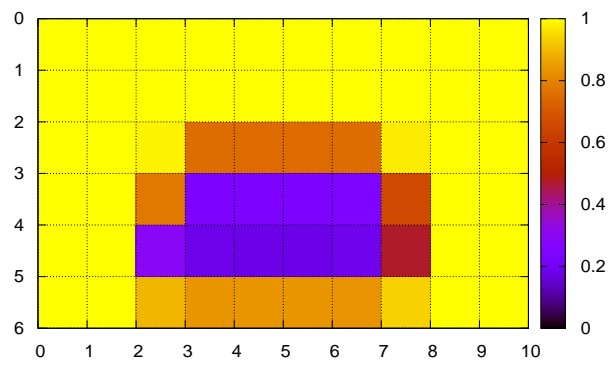

Figura 10: Diagonal principal da matriz de resolução de modelo, caso sobredeterminado, com 50 valores singulares. A escala de cores fornece a amplitude adimensional.

com vetores ou com matrizes de forma que:

$$
\begin{aligned}
\mathbf{m} & \Longleftrightarrow \mathbf{M}, \\
\mathbf{m}^{c} & \Longleftrightarrow \mathbf{M}^{c}, \\
\mathbf{w} & \Longleftrightarrow \mathbf{W},
\end{aligned}
$$

onde

$$
\mathbf{m}+\mathbf{m}^{c}=\mathbf{w},
$$

ou

$$
\mathbf{M}+\mathbf{M}^{c}=\mathbf{W} .
$$

Neste último caso $\mathbf{W}$ é uma matriz constante, dado por $\mathbf{W}=\left(w_{0}\right) ; w_{0} \geq \max \left(m_{i}\right)$. A partir de $\mathbf{d}^{o b s}$ e $G$ determinamos $\mathbf{m}^{\text {est }}$ por qualquer método de inversão, no caso deste trabalho será por SVD. Depois calculamos o vetor complementar dos parâmetros de dado observados usando a relação $\mathbf{d}^{o b s, c}=G \mathbf{w}-\mathbf{d}^{o b s}$. Uma segunda inversão é efetuada obtendo agora o vetor complementar dos parânmetros de modelo $\mathbf{m}^{e s t, c}$. De posse dessas duas soluções, $\mathbf{m}^{e s t}$ e $\mathbf{m}^{e s t, c}$, efetuamos então a soma:

$$
\mathbf{w}^{e s t}=\mathbf{m}^{e s t}+\mathbf{m}^{e s t, c} .
$$


Esse resultado será $\mathbf{w}^{\text {est }}=\mathbf{w}$, se o problema inverso for exato. Como geralmente isso não ocorre, podemos verificar em quais regiões do modelo a inversão teve êxito. Portanto, temos uma clara visão se o método de inversão utilizado foi efetivo ou não, seja no modelo como um todo ou em partes do modelo Basseri, A. (2000).

Na Figura 11 podemos observar os resultados do $\mathbf{m}^{e s t, c}$ para 50 valores singulares, com um valor $\mathbf{w}$ constante e igual a 3. Na Figura 12 mostra-se o resultado da Equação 7, para 50 valores singulares, onde os valores de $\mathbf{w}^{e s t}$, próximos de 3 , representam que a inversão foi efetiva e os valores distantes de 3 indicam que a inversão não foi satisfatória.

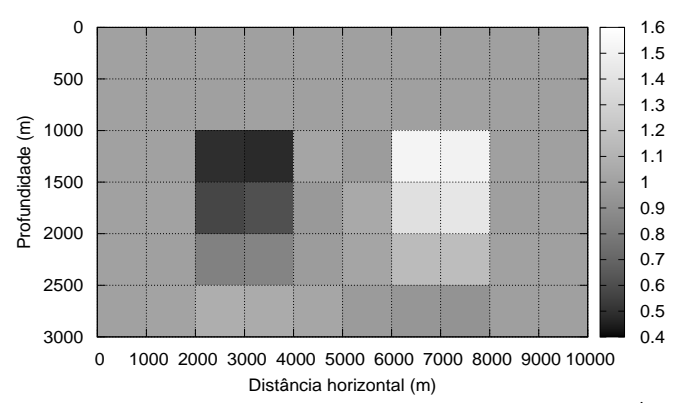

Figura 11: Modelo estimado complementar $\mathbf{m}^{e s t, c}$, no caso determinado, com 50 valores singulares. A escala de cores representa o valor da densidade complementar em $\mathrm{g} / \mathrm{cm}^{3}$.

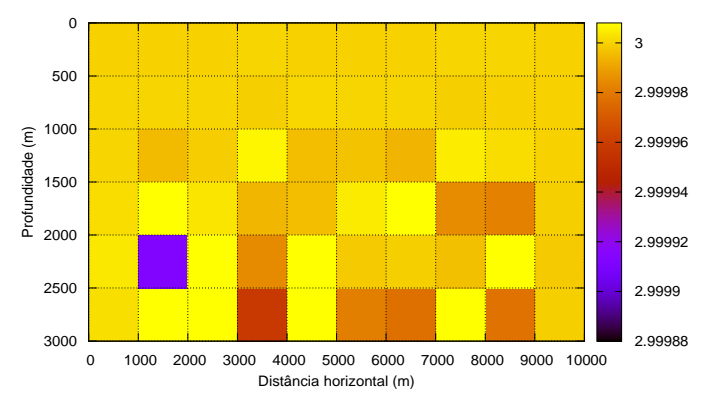

Figura 12: Vetor constante $\mathbf{w}^{e s t}$, caso determinado, com 50 valores singulares. A escala de cores fornece a amplitude adimensional.

\section{CONCLUSÕES}

A discretização de uma região de interesse em blocos 2D retangulares com dimensões conhecidas e com valores de densidade constante em cada um dos blocos, permitiu realizar um estudo detalhado da inversão no modelo testado. Os resultados para diferentes valores singulares permitem observar uma melhoria na qualidade da solução quando é utilizado um número ótimo de valores singulares. Os valores de erro do vetor de parâmetros do modelo $e_{\mathbf{m}}$ e de erro do vetor de parâmetros de dado $e_{\mathbf{d}}$, dependem do número de valores singulares utilizados para a construção da matriz pseudo-inversa. A partir disto constatamos que os menores valores de $e_{\mathbf{m}}$ ocorrem quando utilizamos 50 valores singulares, na situação de dados livres de ruído. Porém, quando contaminamos os dados com ruído gaussiano, verificou-se que o número ótimo de valores singulares é em torno de 22 . Com o emprego da matriz de resolução de modelo, concluimos que o valor da diagonal principal é aproximadamente 1 com a utilização do número ótimo de valores singulares (50 sem ruído e 22 com ruído). Esse comportamento também é observado na matriz de resolução de dado. Além disso, estudamos o comportamento da diagonal principal da matriz de resolução de modelos, de modo a avaliar a qualidade da inversão para cada um dos blocos do meio discretizado, observando-se em cada inversão o valor do $e_{\text {diag }}$ o caso con dados livres de ruído e com dados contaminados com ruído gaussiano. Esses fatos se obteve tanto no caso determinado como também no caso sobredeterminado, como resultado se observo que os menores valores de $e_{\text {diag }}$ ocurrem quando utilizamos um valor ótimo de valores singulares. Com o método de Barbieri, pode-se observar e analisar na solução do problema inverso, isto é, no vetor de parâmetros de modelo estimado, quais regiões a inversão foi efetiva e quais regiões a inversão não foi satisfatória.

\section{AGRADECIMENTOS}

Os autores agradecem ao CNPq (INCT-Geofísica de Petróleo) e à FINEP (Rede CT-PETRO em Geofísica de Exploração) pelo financiamento e apoio à pesquisa. Silvia Bermúdez agradece ao CNPq pela bolsa de doutorado e Amin Bassrei agradece ao CNPq pela bolsa de produtividade de pesquisa.

\section{REFERÊNCIAS}

Barbieri, M., 1974, A criterion to evaluate three dimensional reconstructions from projections of unknown structures, Journal of Theoretical Biology, 48, 451-467.

Bassrei, A., 2000, Novel approaches for the solution and solution evaluation of linear and non-linear inverse problems in geophysics. Boletim SBMAC, Vol. 4.

Bassrei, A., 1993, Regularization and inversion of 2-D gravity data, 63rd Society of Exploration Geophysicists Annual Meeting, 405-409.

Jackson, D. D., 1972, Interpretation of Inaccurate, Insufficient and Inconsistent Data. Geophys. J. R. astr. Soc., 28, 97-109.

Silva, J. e Bassrei, A., 2007, Critérios de seleção de Valores Singulares em Problemas Inversos Lineares: uma Aplicação em Tomografia de Tempos de Trânsito.

Last, B. J. e Kubik, K., 1981, Compact gravity inversion. Geophysics, 48, 713-721. 